\title{
Le récepteur de la thyrotropine : un membre pas comme les autres de la famille des récepteurs couplés aux protéines $\mathbf{G}$
}

Le gène du récepteur de la TSH (thyroid stimulating hormone) a été cloné grâce à ses analogies structurales avec d'autres récepteurs couplés aux G-protéines. Le récepteur de la TSH est assez proche de ceux d'autres hormones glycoprotéiques, la FSH et la LH, partageant avec eux un grand segment extramembranaire comportant un motif caractéristique riche en leucines. L'expression de l'ADNc du récepteur dans des cellules non thyroïdiennes leur confère la propriété de lier la TSH et les auto-anticorps caractéristiques de la maladie de Basedow, ce qui devrait permettre de développer un nouveau dosage biologique de ces anticorps.

\section{Gilbert Vassart Marc Parmentier Frédérick Libert} Jacques Dumont

\begin{abstract}
ADRESSE
G. Vassart : professeur à l'Institut de recherche interdisciplinaire et du service de génétique médicale. M. Parmentier: Chercheur qualifié Fnrs. F. Libert: aspirant Fnrs. J. Dumont : professeur, directeur de l'Institut de recherche interdisciplinaire. Hôpital Érasme, Université libre de Bruxelles, campus Érasme, 808, route de Lennik, 1070 Bruxelles, Belgique.
\end{abstract}

\section{TIRÉS A PART}

G. Vassart.

$\mathrm{m} / \mathrm{s} n^{\circ} 10$, vol. 6 , décembre 90 'hormone hypophysaire thyrotropine ou TSH (thyroid stimulating hormone) est le principal agent régulateur agissant sur la glande thyroïde [1]. Elle contrôle la fonction thyroïdienne (la sécrétion des hormones thyroïdiennes T3 et T4), elle maintient l'expression du phénotype différencié du thyrocyte (synthèse de la thyroglobuline, de la thyroperoxydase et de la pompe à iodure) et elle constitue le principal facteur de croissance de la glande [2]. Dans la majorité des espèces, tous ces effets de la TSH peuvent être reproduits par des agents qui augmentent l'AMP cyclique. Cela n'exclut pas la participation d'autres mécanismes de régulation (telle la cascade des phosphoinositides et du calcium) ou d'autres facteurs de croissance (tel l'epidermal growth factor, EGF). Il est néanmoins bien admis que c'est la modulation par la TSH de la cascade "adénylate cyclase-AMPc protéine kinase A " qui constitue le principal circuit de régulation [1].

L'existence d'un récepteur de la TSH sur la membrane basale du thyrocyte a conduit à l'élaboration d'une théorie étiopathogénique cohérente des hyper- et hypothyroïdies d'origine auto-immunitaire. Dans la maladie de Graves-Basedow, le récepteur est activé par des autoanticorps qui miment l'effet de la TSH et provoquent ainsi l'apparition d'un goitre et d'une hyperthyroïdie ; dans le myxœdème idiopathique, d'autres auto-anticorps, en se liant au récepteur sans l'activer, empêchent la liaison de la TSH et conduisent ainsi à l'involution de la glande et à l'hypothyroïdie [3].

Élément clé dans notre compréhension de la physiologie et de la physiopathologie thyroïdienne, le récepteur de la TSH a été l'objet d'une recherche acharnée visant à l'analyser, à le purifier et, finalement, à en cloner le gène. Bien qu'une image assez pré- 


\section{RÉFÉRENCES}

1. Dumont JE, Vassart G, Refetoff $S$ Thyroid disorders : In : Scriver CR, Beau$\operatorname{det}$ AL, Sly WS, Valle D, eds. The Metabolic Basis of Inherited Diseases (6th edition). New-York McGraw-Hill Inc. 1989; 2: 1843-51.

2. Dumont JE, Jauniaux JC, Roger PP The cyclic AMP-mediated stimulation of cell proliferation. TIBS 1989 ; 14: 67-71.

3. Rees Smith B, McLachlan SM, Furmaniak J. Autoantibodies to the thyrotropin receptor. Endocrine Reviews 1988 ; 9 : 106-20.

4. Parmentier M, Libert F, Maenhaut C, et al. Molecular cloning of the thyrotropin receptor. Science 1989 ; 246 : 1620-2.

5. Loosfelt H, Misrahi M, Atger M, et al. Cloning and sequencing of porcine LH-hCG receptor cDNA : variants lacking transmembrane domain. Science $1989 ; 245: 525-7$.

6. McFarland KC, Sprengel R, Phillips HS, et al. Lutropin-choriogonadotropin receptor : an unusual member of the $G$ protein-coupled receptor family. Science $1989 ; 245$ : 494-9.

7. Nagayama $Y$, Kaufman $K D$, Seto $P$, Rapoport B. Molecular cloning, sequence and functional expression of the cDNA for the human thyrotropin receptor. Biochem Biophys Res Commun 1989 ; 165 : 1184-90.

8. Misrahi M, Loosfelt H, Atger M, Sar S, Guiochon Mantel A, Milgrom E. Cloning sequencing and expression of human TSH receptor. Biochem Biophys Res Commun 1990 ; $166: 394-403$.

9. Lindsay A, Robbins L, Stork $\mathrm{P}$, et al. Isolation of TSH and LH/CG receptor cDNAs from human thyroid : regulation by tissue specific splicing. Mol Endocrinol 1990 ; $4: 1264-76$

10. Akamizu T, Ikuyama S, Saji $M$, et al. Cloning, chromosomal assignment, and regulation of the rat thyrotropin receptor expression of the gene is regulated by thyrotropin, agents that increase cAMP and thyroid anto antibodies. Proc Natl Acad Sci USA 1990 ; 87 : 5677-81.

11. Libert $F$, Ruel J, Ludgate $M$, et al. Thyroperoxidase, an auto-antigen with a mosaic structure made of nuclear and mitochondrial gene modules. EMBO J $1987 ; 6$ : 4193-6.

12. Dixon RAF, Kobilka BK, Stader DJ, et al. Cloning of the gene and cDNA for mammalian beta-adrenergic receptor and homology with rhodopsin. Nature 1986 ; $321: 75-9$.

13. Kubo T, Fukuda K, Mikami A, et al. Cloning, sequencing and expression of complementary DNA encoding the muscarinic acetylcholine receptor. Nature $1986 ; 323$ : 411-6.

14. Birnbaumer L, Abramowitz J, Brown AM. Receptor-effector coupling by G proteins. Biochim Biophys Acta 1989 ; cise du récepteur ait été obtenue par des protéines [3], les essais de purification et de clonage ont été pendant longtemps marqués par la frustration, l'échec et les fausses nouvelles pas toujours démenties. Ce long vide de la période "d'avant le clonage" a maintenant fait la place à une surabondance ; peu après notre publication initiale [4], plusieurs groupes, exploitant la percée réalisée par les équipes de Milgrom [5] et de Segaloff-Seeburg [6] (le clonage du récepteur de la $\mathrm{LH}$ ), rapportaient le clonage du récepteur de la TSH [7-10].

\section{Stratégies de clonage}

Dans cette longue course au récepteur, un éventail de stratégies a été utilisé. La première et la plus évidente a consisté à cribler des banques d'expression d'ADNc thyroïdiens au les techniques classiques de la chimie moyen d'auto-anticorps (de patients atteints de maladie de GravesBasedow ou de myxœdème idiopathique) ou d'anticorps monoclonaux supposés reconnaître spécifiquement le récepteur de la TSH. Cette approche - qui avait porté ses fruits pour le clonage d'un autre auto-antigène thyroïdien, la thyroperoxydase [11] - échoua. Elle conduisit, néanmoins, au clonage d'une série d'" auto-antigènes " nouveaux dont certains se révèleront peut-être intéressants dans le futur.

Les études séminales des groupes de Lefkowitz-Dixon [12] et de Numa [13], démontrant que les récepteurs couplés aux protéines $\mathrm{G}$ constituent une famille évolutive ayant un ancêtre commun avec celle des pigments visuels, allaient ouvrir la voie à une stratégie nouvelle et fructueuse. Les gènes de cette famille codent pour des protéines présentant

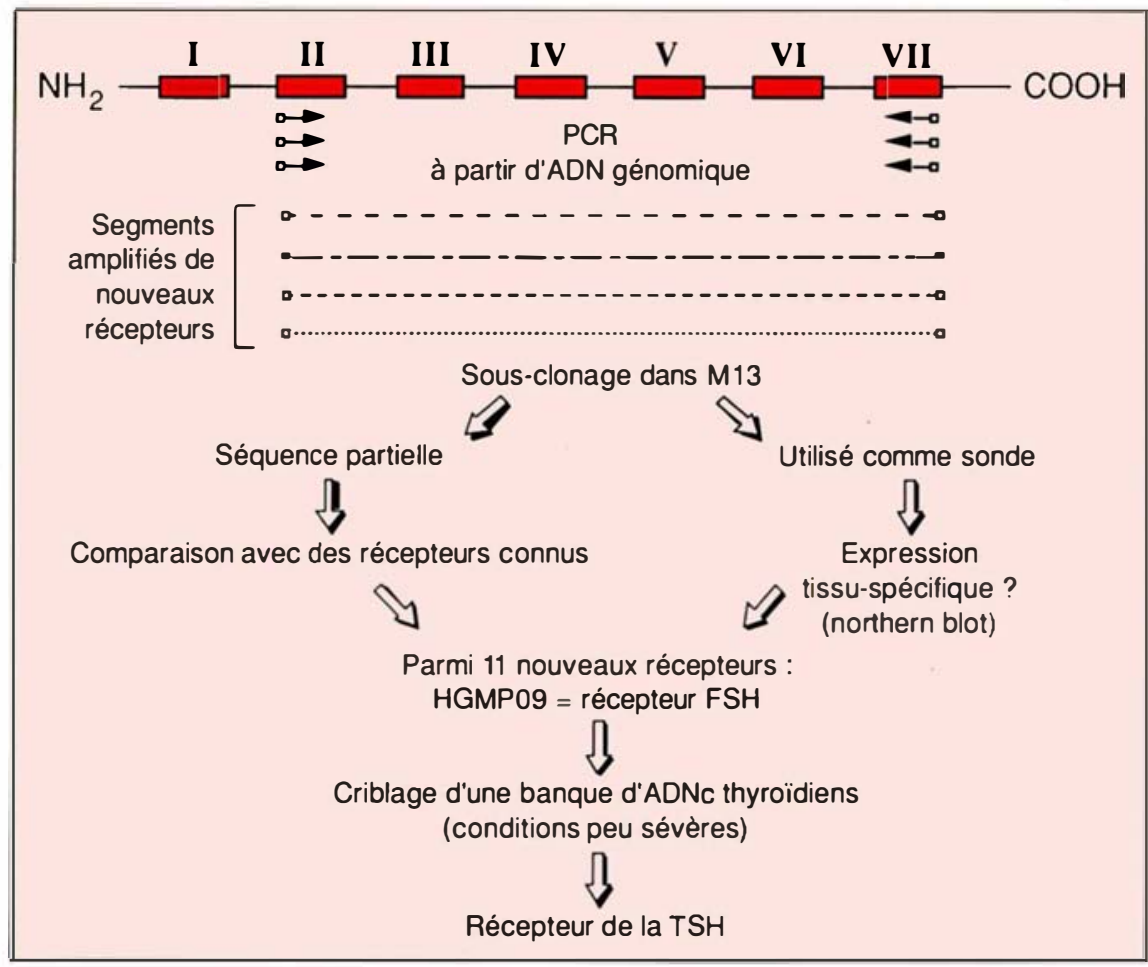

Figure 1. Stratégie ayant conduit au clonage du récepteur de la TSH. Un récepteur type appartenant à la famille des récepteurs couplés aux protéines $G$ est schématisé par la ligne supérieure portant sept rectangles rouges qui indiquent la position des sept segments transmembranaires (en chiffres romains). Les couples de flèches horizontales représentent les amorces "dégénérées" utilisées pour amplifier par PCR des segments de nouveaux récepteurs entre les domaines transmembranaires II et VII. 
sept hélices transmembranaires. Les hormones hypophysaires glycoprotéiques TSH, LH et FSH étant connues pour agir par l'intermédiaire de la protéine $\mathrm{G}_{\mathrm{s}}$ [14], il était logique de postuler que leurs récepteurs appartiendraient à la même famille multigénique. L'alignement des structures primaires des différents membres de cette famille fait apparaître une très grande conservation, en particulier dans les hélices transmembranaires. Cela suggéra qu'il devait être possible d'exploiter une approche fondée sur la réaction en chaîne de la polymérase (PCR) [15] pour cloner de nouveaux récepteurs couplés aux protéines G. La stratégie consistait à utiliser une paire d'amorces "dégénérées " définies par les séquences consensus des $\mathrm{ADNc}$ qui codent pour les zones les plus conservées des hélices transmembranaires III et VI des récepteurs connus [16] (figure 1, p. 986).

Si la méthode conduisit au clonage de quatre nouveaux récepteurs putatifs, le récepteur de la TSH n'était pas du nombre [16]. On sait maintenant que les récepteurs des hormones glycoprotéiques different du consensus au niveau de l'extrémité 3' de la troisième zone transmembranaire (voir plus loin). Néanmoins, la méthode avait démontré son efficacité (un des récepteurs clonés de cette manière constitue un nouveau soustype de récepteur à la sérotonine ; un autre est un marqueur spécifique du striatum, mais son ligand reste mystérieux [17]) et se prêtait à des variantes. En utilisant la même stratégie avec une paire d'amorces dégénérées correspondant cette fois à des segments des zones transmembranaires II et VII, une série de récepteurs putatifs furent clonés en partant d'ADN génomique humain. Parmi ceux-ci, il en était un (appelé HGMP09 dans l'attente d'une identification précise) qui présentait les caractéristiques attendues d'un récepteur de gonadotrophines [4]. Utilisé comme sonde dans des hybridations à basse stringence, HGMP09 conduisit à isoler d'une banque d'ADNc thyroïdiens canins un clone présentant toutes les caractéristiques attendues du récepteur de la TSH [4]. L'homologue humain fut dès lors cloné facilement [18]. La comparai-

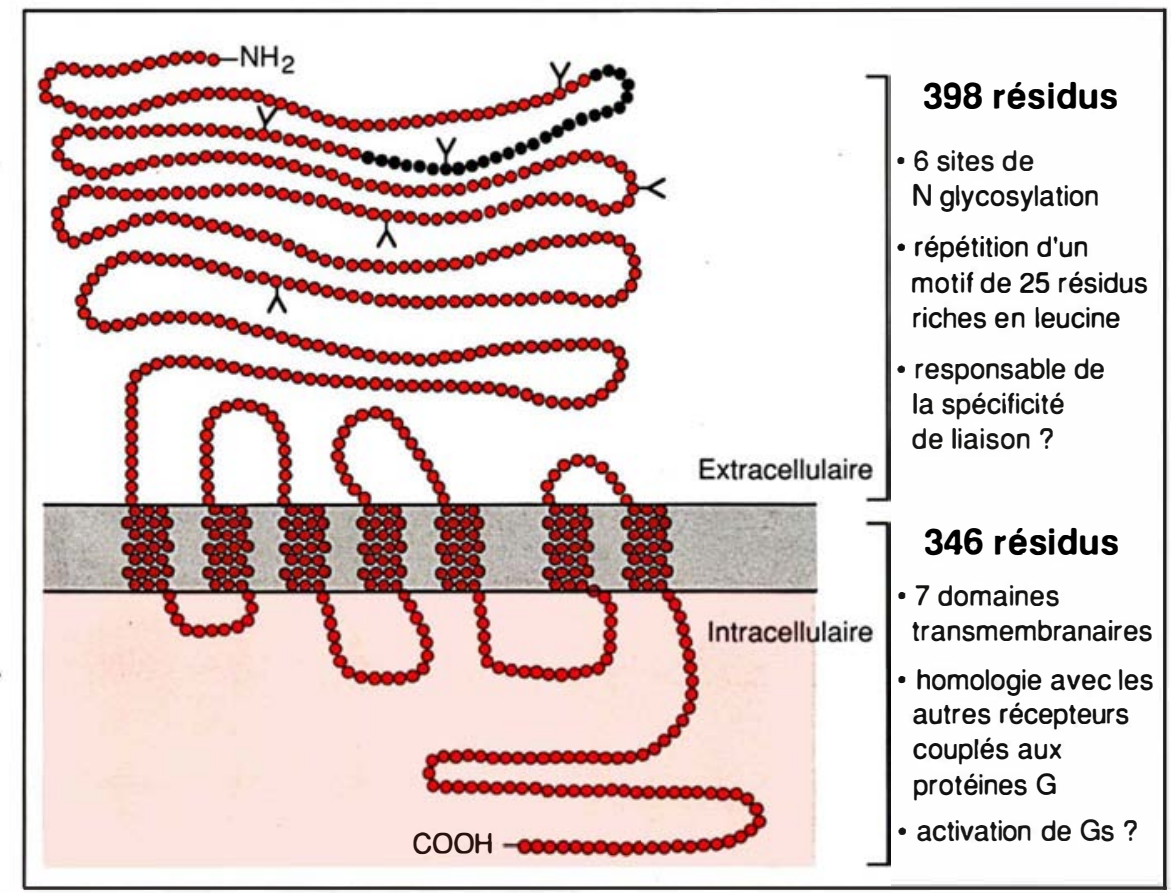

Figure 2. Représentation schématique du récepteur humain de la TSH. Les symboles $Y$ représentent les sites potentiels de glycosylation. Le segment extracellulaire représenté en noir correspond à une unité de répétition riche en leucine (voir texte); ce segment est celui dont l'absence a été démontrée dans un variant du récepteur identifié chez le chien.

son des séquences de HGMP09 et du récepteur de la TSH avec celle du récepteur de la $\mathrm{LH}$ révéla que ces trois molécules constituaient une sous-famille des récepteurs couplés aux protéines $\mathrm{G}$ présentant des caractéristiques distinctes (voir ci-dessous). Notre suggestion selon laquelle HGMP09 correspondrait au récepteur de la $\mathrm{FSH}$ a été confirmée récemment [19].

L'homologie de séquence attendue entre les récepteurs des hormones glycoprotéiques a été exploitée par plusieurs groupes pour cloner le récepteur de la TSH en utilisant comme sonde la séquence du récepteur de la $\mathrm{LH}$. Il résulte de ces travaux que le récepteur de la TSH est maintenant disponible dans au moins trois espèces : l'homme, le chien et le rat $[4,18,7-10]$.

\section{Structure du récepteur de la TSH}

Les récepteurs de la TSH, de la $\mathrm{LH}$ et de la FSH possèdent en commun une caractéristique qui les distingue des autres récepteurs couplés aux protéines $\mathrm{G}$ : en plus du segment carboxyl-terminal qui comporte les sept domaines transmembranaires (346 résidus pour le récepteur de la TSH), ils présentent un segment extracellulaire amino-terminal de longueur comparable (398 résidus pour le récepteur de la TSH humaine) précédé d'un peptide signal (figure 2). Ce domaine contient des sites accepteurs pour la $\mathrm{N}$-glycosylation (six pour le récepteur de la TSH humaine) et est constitué de la répétition très imparfaite d'un motif de vingt-cinq résidus, riche en leucines [6]. Ce motif - qui est retrouvé dans une série de protéines aux fonctions diverses - présenterait une structure d'hélices amphiphile qui lui conférerait l'aptitude d'interagir avec d'autres protéines. L'alignement des structures primaires des trois récepteurs démontre une conservation plus grande de leurs domaines transmembranaires (environ $70 \%$ ) que de leurs extensions extracellulaires (environ $40-45 \%$, figure 3 , p. 988). Cela conduit tout naturellement à propo- 


\section{RÉFÉRENCES}

15. Saiki RK, Gefland DH, Stoffel S, et al. Primer-directed enzymatic amplification of DNA with a thermostable DNA polymerase. Science $1988 ; 239$ : 487-91.

16. Libert F, .Parmentier M, Lefort A, et al. Selective amplification and cloning of four new members of the $G$ protein-coupled receptor family. Science 1989 ; 244 : 569-72.

17. Schiffmann SN, Libert F, Vassart G, Dumont JE, Vanderhaeghen JJ. A cloned $G$ protein-coupled protein with a distribution restricted to striatal medium-sized neurons. Possible relationship with D1 dopamine receptor. Brain Research 1990 ; 519 : 333-7.

18. Libert F, Lefort A, Gérard C, et al. Cloning, sequencing and expression of the human thyrotropin receptor: evidence for binding of autoantibodies. Biochem Biophys Res Commun 1989; 165 : 1250-5.

19. Sprengel R, Braun $T$, Nikolics $K$, Segaloff D, Seeburg PH. The testicular receptor for follicle stimulating hormone : structure and functional expression of cloned cDNA. Mol Endocrinol 1990; 4 : 525-30.

20. Strader CD, Sigal IS, Dixon RA, et al. Structural basis of beta adrenergic receptor function. FASEB J $1989 ; 3: 1825-32$.

21. Libert F, Parmentier M, Majenhaut C, et al. Molecular cloning of a dog thyrotropin (TSH) receptor variant. Mol Cell Endocrinol $1990 ; 68$ : R15-R17.

22. Libert F, Passage E, Lefort A, Vassart G, Mattei MG. Localization of human thyrotropin receptor gene to the q31 band of chromosome 14 by in situ hybridization. Cytogen Cell Genet 1990 ; (sous presse).

23. Laurent E, Mockel J, Van Sande J, Graff I, Dumont JE. Dual activation by TSH of phospholipase-C and cAMP cascades in human thyroid. Mol Cell Endocrinol $1987 ; 52: 273-5$.

24. Perret J, Ludgate $M$, Libert F, et al. Stable expression of the human TSH receptor in $\mathrm{CHO}$ cells and characterization of differentially expressing clones. Biochem Biophys Res Commun 1990 ; 171 : 1044-50.

25. Ludgate $\mathbf{M}$, Perret J, Parmentier $\mathbf{M}$, et al. Use of the recombinant human thyrotropin receptor (TSHr) expressed in mammalian cell lines to assay TSHr autoantibodies. Mol Cell Endocrinol 1990; 73 : R13-18.

26. McKenzie JM, Zakarija M. The clinical use of thyrotropin receptor antibody measurements. J Clin Endocrinol Metab 1989 ; $69: 1093-6$

27. Higgins DG, Sharp PM. A package for performing multiple sequence alignments on a microcomputer. Gene $1988 ; 73: 237-44$.

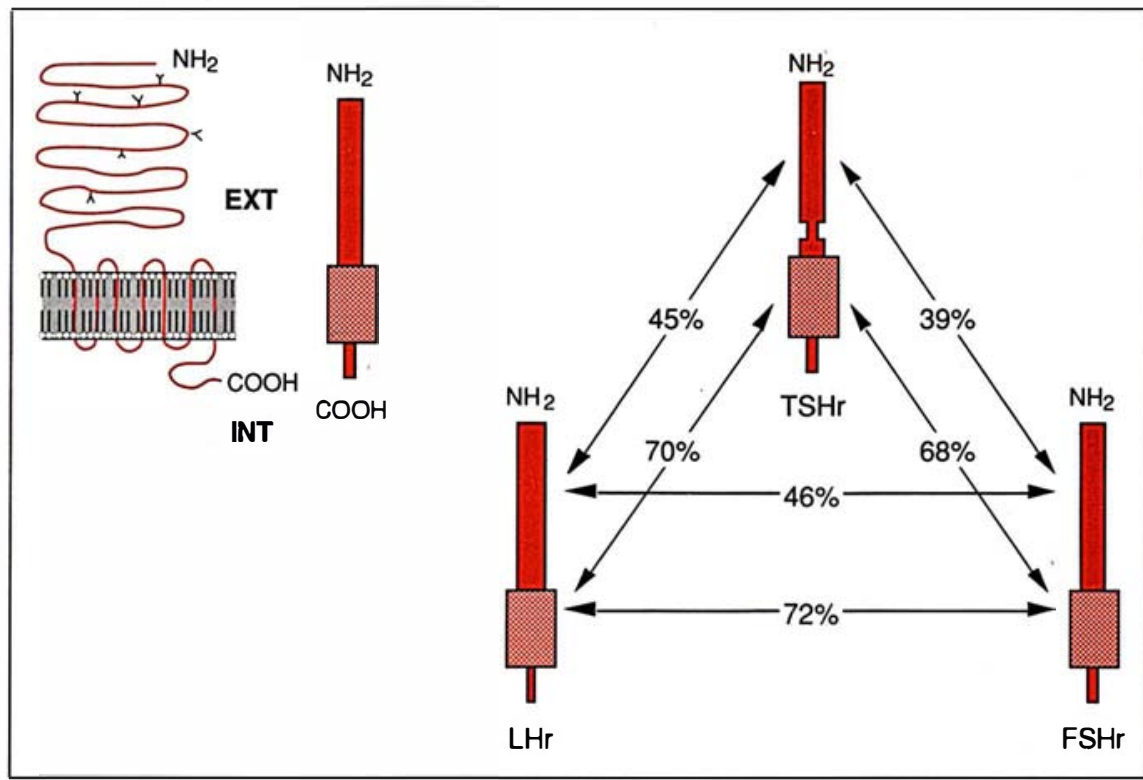

Figure 3. Homologie de structure primaire entre les différents récepteurs des hormones glycoprotéiques. Les similitudes de séquence sont indiquées séparément pour les domaines extracellulaires amino-terminaux et pour la moitié carboxyl-terminale des récepteurs qui contient les domaines transmembranaires. Le segment de 51 acides aminés qui est propre au récepteur de la TSH est indiqué par une encoche à la lisière entre le domaine extracellulaire et le premier segment transmembranaire.

ser que la spécificité de liaison entre le récepteur et la sous-unité $\beta$ des hormones glycoprotéiques serait prise en charge par le domaine extracellulaire, alors que l'activation de la protéine $G_{s}$ serait réalisée par le domaine carboxyl-terminal. En particulier, la troisième boucle intracellulaire, qui a été impliquée dans l'interaction avec les protéines $\mathrm{G}$ des récepteurs adrénergiques et muscariniques [20], présente une conservation parfaite dans sa partie carboxyl-terminale.

Une particularité propre au récepteur de la TSH est la présence d'un segment d'une cinquantaine de résidus situé près de la jonction entre le domaine extracellulaire et le premier segment transmembranaire. L'avenir nous dira si ce segment joue un rôle dans la production d'éventuelles formes solubles du récepteur (zone de clivage ?). L'existence de telles formes est suspectée pour le récepteur de la LH, sur la base de transcrits dont l'épissage différentiel raccourcit le cadre de lecture au seul domaine extracellulaire [5]. A ce jour, bien que deux transcrits soient présents dans la thyroïde humaine $(4,6$ et $4,4 \mathrm{~kb})$ [18], il n'existe pas d'évidence expérimentale en faveur d'un épissage différentiel similaire pour le récepteur de la $\mathrm{TSH}$.

La stratégie qui nous a permis de cloner un segment du récepteur de la FSH ainsi que des expériences non publiées sur l'ADN chromosomique du récepteur de la TSH humain nous indiquent que la plus grande partie de la région transmembranaire de ces récepteurs est codée par un seul exon. Au contraire, on peut s'attendre à ce que l'information génétique qui code pour le domaine extracellulaire du récepteur soit morcelée en un grand nombre d'exons. Cette hypothèse, suggérée par la nature répétitive de cette région de la protéine, est renforcée par la description d'un variant du récepteur de la TSH chez le chien, auquel manque le second motif riche en leucine [21] (figure 2). Cette anomalie est probablement la conséquence d'une imprécision physiologique de l'épissage ou d'un polymorphisme associé à une mutation dans un site d'épissage. Le gène du récepteur humain 
de la TSH est situé sur le bras long du chromosome 14 [22].

En plus de leur domaine extracellulaire de grande taille, les récepteurs des hormones glycoprotéiques présentent des caractéristiques qui les distinguent des autres récepteurs couplés aux protéines $G$. D'une façon générale, l'alignement des segments transmembranaires des différents membres de cette grande famille évolutive permet de construire un dendrogramme qui fait ressortir l'appartenance des récepteurs $\mathrm{TSH}, \mathrm{LH}$ et $\mathrm{FSH}$ à une sous-famille distincte (figure 4). Parmi les différences notables, le tripeptide canonique "DRY " (Asp Arg Tyr) est remplacé par la séquence "ERW" (Glu Arg Trp) à la frontière entre le troisième segment transmembranaire et la deuxième boucle intracellulaire. Notons également l'absence de résidu aspartate à la position correspondant à l'acide aminé 113 du récepteur ßadrénergique dans ce même troisième domaine transmembranaire. $\mathrm{Ce}$ résidu est retrouvé dans tous les récepteurs dont les ligands sont des amines et a été impliqué dans la formation d'une liaison ionique entre le ligand et la poche constituée au sein de la membrane plasmique par les hélices transmembranaires [20].

\section{Expression du récepteur dans des cellules non thyroiddiennes}

Le clonage du récepteur de la TSH permet d'explorer sa fonction dans un environnement différent de celui du thyrocyte. Bien que préliminaires, les expériences réalisées avec les récepteurs humain et canin permettent déjà de tirer les conclusions suivantes. L'expression du récepteur dans des systèmes hétérologues par microinjection d'ARN recombinant ou par transfection, confere à ces systèmes la propriété de lier la TSH marquée (cellules $\mathrm{COS}$ ou $\mathrm{CHO}$ ) ou d'accumuler de l'AMP cyclique en réponse à la $\mathrm{TSH}$ (cellules Y1, cellules $\mathrm{CHO}$, oocytes de xénopes) [4, 18]. Des expériences préliminaires ont permis de montrer que l'effet combiné de la TSH - observé dans l'espèce humaine sur les cascades de l'AMP cyclique et des phospho-inositides [23] - peut être reproduit dans des cellules $\mathrm{CHO}$ exprimant le seul récepteur $\mathrm{m} / \mathrm{s} n^{\circ} 10$, vol. 6 , décembre 90

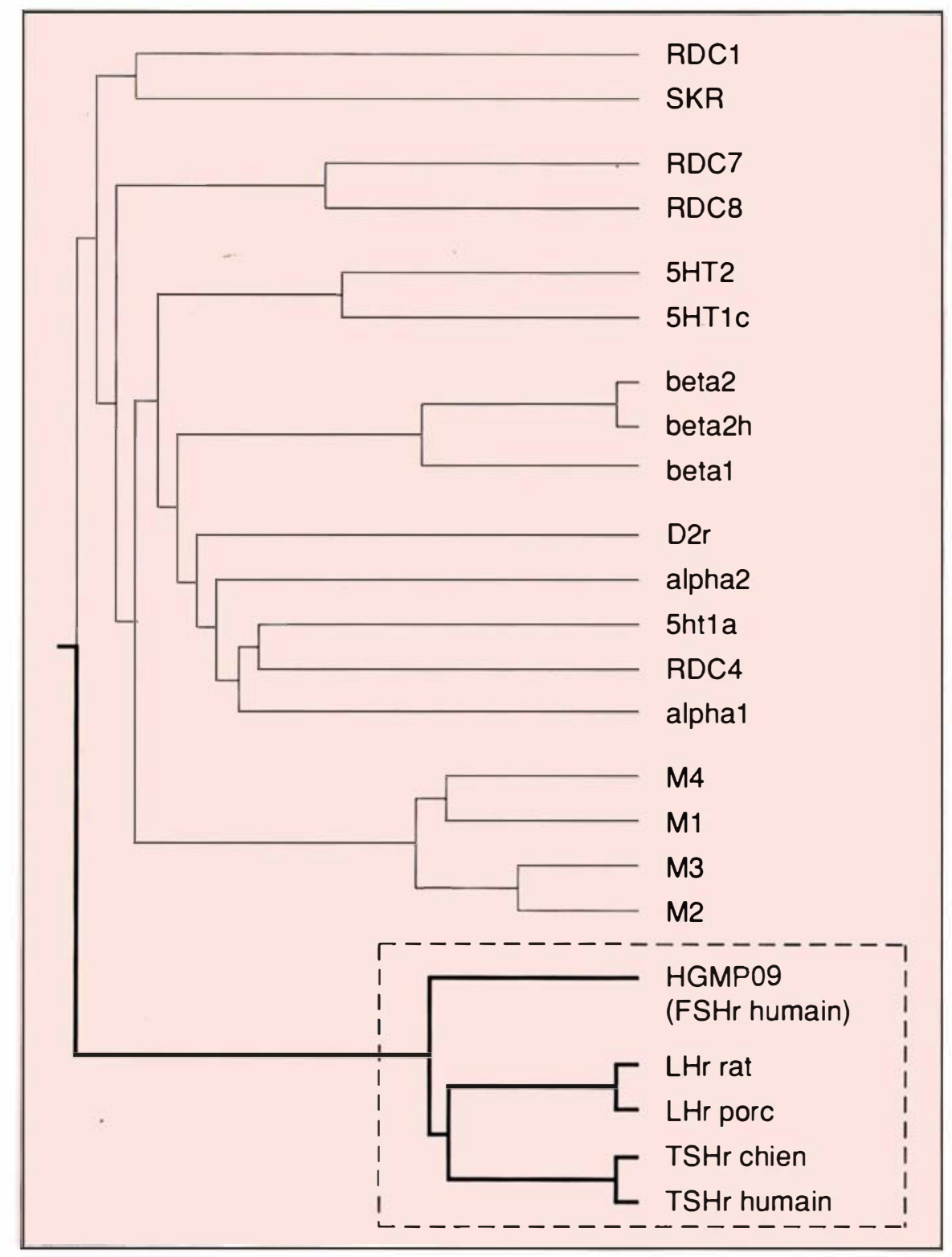

Figure 4. Représentation en dendrogramme d'un échantillon représentatif de la superfamille des récepteurs couplés aux protéines G. Le programme CLUSTAL a été utilisé [27]. La longueur des segments horizontaux est proportionnelle aux scores de similitude calculés à partir d'un alignement de séquences partielles (c'est-à-dire de segments qui ont pu être alignés de façon non ambiguë, sans qu'il soit nécessaire d'introduire de " trous " importants; pour détails et références concernant les séquences reprises dans le dendrogramme, consulter Libert et al., 1989). SKR : récepteur de la substance $K$; $5 H T 1 a, 1 c, 2$ : récepteur de la sérotonine des différents sous-types ; $\beta$ 1,2 ; récepteur $\beta$ adrénergiques ; $\beta$ 2h : récepteur $\beta$ adrénergique de hamster ; $\alpha$ 1, 2 : récepteur $\alpha$ adrénergiques; $D 2 r$ : récepteur dopaminergique du type D2: M1, M2, M3, M4: récepteurs muscariniques des sous-types correspondants ; $R D C 1, R D C 4, R D C 7, R D C 8$ : récepteurs "orphelins " en attente de l'identification de leurs ligands. 
actuellement disponible sous forme de clone (Van Sande et al., non publié). La recherche d'autres sous-types de récepteurs ne semblerait donc pas se justifier. Le même type d'expérience devrait également nous apprendre si le comportement du récepteur est, ou non, influencé par son environnement dans la membrane du thyrocyte.

Il y a peu de doute que le récepteur de la TSH soit l'antigène majeur impliqué dans la maladie de GravesBasedow. Néanmoins, la contribution d'autres composants de la membrane plasmique a été évoquée. L'expression du récepteur cloné dans des cellules non thyroïdiennes [24] permettra de déterminer si cela leur confere la propriété de répondre aux différents types d'auto-anticorps décrits à ce jour. Des résultats préliminaires démontrent que la TSH radio-marquée est très efficacement déplacée du récepteur exprimé dans des cellules COS par des immunoglobulines de patients atteints de myxœdème idiopathique [18]. De même, des cellules $\mathrm{CHO}$ transfectées de façon stable par l'ADNc du récepteur humain répondent par une augmentation sensible de leur AMP cyclique intracellulaire à l'addition d'immunoglobulines de patients souffrant de la maladie de GravesBasedow [25]. On peut s'attendre que de telles lignées cellulaires à croissance rapide remplacent prochainement les cellules de rat (FRTL5) ou les cellules humaines dans les dosages biologiques [26] utilisés pour mesurer les immunoglobulines douées de propriétés thyréostimulantes.

La production de récepteur recombinant de la TSH sur un " terrain " non thyroïdien facilitera grandement la préparation d'anticorps monoclonaux. Cette approche conduira à définir la carte des épitopes de la molécule et à identifier, parmi eux, les auto-épitopes impliqués dans son activation ou son inhibition par des auto-anticorps. Le criblage au moyen d'auto-anticorps d'une bibliothèque d'expression contenant tous les épitopes linéaires possibles du récepteur indique que les épitopes impliqués dans la pathologie sont principalement de nature conformationnelle (Libert et al., non publié). Cela fournit a posteriori une explication aux échecs des stratégies de clonage fondées sur le criblage de banques d'expression par des auto-anticorps.
Lorsqu'ils seront disponibles, les anticorps monoclonaux anti-récepteur de la TSH constitueront des outils puissants pour explorer les mécanismes d'(in)activation du récepteur. Utilisés comme sonde dans des expériences de compétition (avec la TSH ou avec des auto-anticorps stimulants ou bloquants), ils permettront d'identifier les segments du récepteur impliqués dans la transduction du signal. De plus, ils conduiront à la mise au point d'une nouvelle génération de tests diagnostiques permettant la détection des auto-anticorps. Il reste néanmoins à démontrer que la glycosylation du récepteur TSH s'effectue normalement dans ces lignées cellulaires non thyroïdiennes.

\section{Perspectives}

Le clonage quasi simultané des récepteurs des trois hormones glycoprotéiques permettra une étude détaillée de leur relations "structure-fonction ". Il ne fait pas de doute que des récepteurs chimériques sont dès à présent construits dans de nombreux laboratoires et que leur étude conduira à définir les domaines protéiques responsables de la spécificité des interactions hormone-récepteur. De même, la comparaison avec les autres membres de la superfamille des récepteurs couplés aux protéines $\mathrm{G}$ et des expériences de mutations dirigées nous révèleront les domaines responsables des interactions avec ces protéines $G$ et de leur activation.

Bien que l'existence de maladies génétiques impliquant le récepteur de la TSH n'ait pas été documentée, on peut prédire une recherche active d'allèles du récepteur potentiellement associés à diverses maladies thyroïdiennes. De plus, il sera maintenant possible de vérifier l'existence d'hypothyroïdies congénitales dues à un défaut du récepteur (Dumont $e t a l$, 1989).

Le clonage du récepteur de la TSH ouvre également la voie à la création de modèles animaux de la maladie de Graves-Basedow (par simple immunisation ou par la méthodologie des souris transgéniques) et, si l'on accepte de rêver quelque peu, au développement de stratégies thérapeutiques fondées sur l'utilisation de peptides synthétiques bien choisis

\section{Summary}

The thyrotropin (TSH) receptor, an unusual member of the G protein coupled receptor family

A major autoantigen involved in thyroid diseases, the thyrotropin receptor has long resisted cloning by classical approaches. Its cDNA has now successfully been cloned by strategies relying on its belonging to the superfamily of $\mathrm{G}$ protein-coupled receptors and its gene has been localized on the long arm of chromosome 14 . The receptor has in common with the other members of this family seven putative transmembrane domains displaying sequence conservation. In addition, it shares with the other recently cloned glycoprotein hormone receptors (LH and FSH) a characteristically long aminoterminal domain made of " leucine-rich " repeated motifs. The glycoprotein hormone receptors show high sequence similitary in their membrane spanning domains (ca. $70 \%$ ), whereas their extracellular domains are more divergent (ca. $45 \%$ ). This suggests that it is the latter which is responsible for hormone binding. Expression of the TSH receptor in non thyroid cells leads to the development of new assays for autoantibodies responsible for Graves disease.

\section{Remerciements}

Cette revue est l'occasion de remercier les nombreuses personnes qui ont participé à la recherche conduisant au clonage du récepteur de la TSH dans notre laboratoire : Daniel et Christiane Christophe, Christiane Dinsart, Catherine Gérard, Anne Lefort, Marian Ludgate, Carine Maenhaut, Claude Massaer, Jason Perret, Marie-Jeanne Simons et Jacqueline $V$ an Sande. Cette recherche a bénéficié du soutien du ministère de la Politique scientifique (sciences de la vie), du Fonds national de recherche scientifique, du Fonds de la recherche scientifique médicale, du National Institute of Health et de l'Association recherche biomédicale et diagnostic. 